\title{
Factores de riesgo asociados a complicaciones anestésicas en enfermos renales crónicos intervenidos de urgencia
}

\author{
Risk factors associated with anesthetic complications in \\ chronic renal patients undergoing emergency surgery \\ Yuselis Benítez Rondón', Kenia Dalia León Paz², C. Yurisnel Ortiz Sánchez, \\ Guillermo Capote Guerrero ${ }^{4}$, Isrrael Antonio Tarancón Serrano ${ }^{5}$.
}

\begin{abstract}
Introduction: Chronic kidney disease is a clinical entity secondary to the common final pathway of multiple etiologies with risk factors common to other chronic diseases. Objective: To identify the risk factors related to the appearance of anesthetic complications in chronic renal patients undergoing emergency surgery. Method: A prospective cohort study was performed in patients with chronic kidney disease undergoing emergency surgery. The exposed cohort consisted of 15 patients with chronic kidney disease who developed complications during the study period. Results: Age over 40 years, patients with ischemic heart disease and hypertensive patients presented a higher risk of anesthetic complications. Obesity and bronchial asthma were no risk factors. It was demonstrated that when the time elapsed between hemodialysis and the operation was less than 2 hours, the risk of anesthetic complications increased, the stage of the disease and the associated drugs were not related to the appearance of complications. Patients who received general anesthesia had three times more risk of complications than those under regional anesthesia. Conclusions: Age, arterial hypertension, ischemic heart disease, heart failure and the use of general anesthesia are risk factors associated with complications.
\end{abstract}

\section{Key words:}

Chronic renal failure, anesthetic complications

Especialista de primer grado en Medicina General Integral y Anestesiología y Reanimación. Instructor.

2 Especialista de primer grado en Medicina General Integral y Anestesiología y Reanimación. Máster en urgencias médicas. Profesor asistente.

Licenciado en farmacología. Profesor Titular.

Especialista de $2^{\text {do }}$ grado en anestesiología y reanimación. Diplomado en terapia intensiva. Profesor asistente.

Especialista de primer grado en anestesiología y reanimación. Profesor asistente.

Fecha de recepción: 20 de mayo de 2018

Fecha de aceptación: 16 de septiembre de 2018

\section{ORCID}

https://orcid.org/0000-0003-2841-8348

Correspondencia:

Guillermo Capote Guerrero

Email: gcapote@infomed.sld 


\section{RESUMEN}

Introducción: La enfermedad renal crónica es una entidad clínica secundaria a la vía final común de múltiples etiologías con factores de riesgo comunes a otras enfermedades crónicas. Objetivo: Identificar los factores de riesgo relacionados con la aparición de complicaciones anestésicas en enfermos renales crónicos intervenidos de urgencia. Método: Se realizó un estudio de cohorte prospectivo en pacientes con enfermedad renal crónica intervenidos por cirugía general de urgencia. La cohorte expuesta estuvo constituida por 15 pacientes con enfermedad renal crónica que desarrollaron complicaciones en el período en estudio. Resultados: La edad mayor o igual a 40 años, los pacientes con cardiopatía isquémica y los hipertensos presentaron mayor riesgo de complicaciones anestésicas, no así la obesidad al igual que el asma bronquial. Se demostró que cuando el tiempo transcurrido entre la hemodiálisis y la operación fue inferior a 2 horas se incrementó el riesgo de complicaciones anestésicas, el estadio de la enfermedad y los medicamentos asociados no estuvieron relacionados con la aparición de complicaciones. Los pacientes a los que se administró anestesia general tuvieron tres veces más riesgo de complicaciones que aquellos que fueron intervenidos con anestesia regional. Conclusiones: La edad, la hipertensión arterial, la cardiopatía isquémica, la insuficiencia cardiaca y el empleo de anestesia general son factores de riesgo asociados a complicaciones, en los pacientes portadores de IRC, sometidos a cirugía de urgencia.

\section{Palabras clave:}

Enfermos renales, complicaciones anestésicas, riesgo

\section{Introducción}

L

a enfermedad renal crónica (ERC) es una entidad clínica bien identificada, secundaria a la vía final común de múltiples etiologías con factores de riesgo comunes a otras enfermedades crónicas no trasmisibles (ECNT), con una característica particular: la existencia de la terapia renal de reemplazo (TRR) o sustitutiva (TRS) por diálisis o trasplante renal ante el daño funcional renal irreversible[1].

En los países desarrollados la tasa de prevalencia es aproximadamente entre 500 a 1.400 pacientes por millón de habitantes. La incidencia (nuevos pacientes que ingresan a tratamiento sustitutivo) es aproximadamente 300 a 340 pacientes por millón de habitantes y se incrementa alrededor de un $10 \%$ anualmente. En los países en vías de desarrollo ambas tasas son mucho menores y está en correspondencia con su nivel económico[2]-[6].

En Latinoamérica la prevalencia es de 447 pacientes por millón de habitantes, y la incidencia es de 147 pacientes por millón de habitantes. En Cuba la prevalencia es de 225 pacientes por millón de habitantes[1].

En varias ocasiones los pacientes con enfermedad renal crónica son llevados a cirugías relacionadas con su diálisis con operaciones propias de sus comorbili- dades[7], por lo que resulta importante destacar las diferentes situaciones que este paciente impone al anestesiólogo:

1) Los problemas y las contingencias clínico-farmacológicas que el paciente renal crónico plantea: derivados de la clínica de la uremia, que impacta en todos los órganos de la economía.

2) Las alteraciones farmacocinéticas, farmacodinamias y toxicológicas como consecuencia de la insuficiencia del órgano responsable de la eliminación de los fármacos o sus metabolitos[8].

La cirugía urgente en pacientes con enfermedad renal crónica se asocia con mayor morbimortalidad, The National Kidney Foundation recomienda indagar durante la evaluación de los pacientes con enfermedad renal crónica: el diagnóstico etiológico, las comorbilidades, la severidad (nivel de función renal), las complicaciones y estimar el riesgo de pérdida de la función renal y de complicación cardiovascular. En la valoración preanestésica estos parámetros deben sumarse a los riesgos inherentes del procedimiento quirúrgico con el fin de optimizar los factores de riesgo[7].

El objetivo principal fue identificar los factores de riesgo que podrían relacionarse con complicaciones anestésicas en pacientes con IRC sometidos a cirugía de urgencia. 


\section{Diseño metodológico}

\section{Tipo y diseño general del estudio}

Se realizó un estudio longitudinal prospectivo de cohorte en pacientes con enfermedad renal crónica operados de urgencia en el Hospital provincial "Carlos Manuel de Céspedes de Bayamo, procedentes de la región del Cauto durante el período comprendido desde el $1^{\text {ro }}$ de octubre de 2014 hasta el 31 de diciembre de 2017.

Estableciendo una prevalencia del factor de exposición en el grupo no enfermo del 33\% y en el grupo enfermo del $23 \%$ con un $95 \%$ de coeficiente de confianza que es igual a un 0,05 de nivel de significación del error, con una potencia de $80(1-\beta)$.

Se estableció que la cohorte expuesta estuvo constituida por los 15 pacientes con enfermedad renal crónica que desarrollaron complicaciones en el período de estudio y cumplieron con los criterios de inclusión. Se les explicó y llenó el consentimiento informado al paciente y al familiar.

Para el cálculo del tamaño de la muestra se utilizó el paquete estadístico Epilnfo versión 2002 para Windows.

Los criterios de exclusión de pacientes en la cohorte fueron los que rechazaron participar y los portadores de enfermedades psiquiátricas o terminales.

\section{Definición operacional de las variables}

Se delimitaron como variables independientes o explicativas del estudio a los factores hipotéticamente asociados al desarrollo de complicaciones anestésicas, que serán sometidos a observación. Como variable dependiente se consideró la aparición o no de complicaciones anestésicas.

Se consideraron, para definir a un paciente con complicaciones anestésicas[9]-[11], los criterios siguientes:

- Cardiacas: taquicardia o bradicardia; hipotensión e hipertensión; aparición de arritmias malignas, trombosis venosa profunda, isquemia miocárdica aguda y paro cardiaco.

- Respiratorias: Hipercapnia e hipocapnia; barotrauma; broncoaspiración; neumomediastino; neumotórax, hipoxia mantenida, aumento de la P1 (presión pico), (P2) presión meseta, embolismo gaseoso.

Como variables independientes para los factores de riesgo quirúrgico en función del enfermo, incluidas en el objetivo específico 1, se considerarón:
- Edad: fue tratada como una variable cuantitativa discreta. Se tomó en valores absolutos de años cumplidos. Se convirtió en dicotómica para el análisis univariado, en dos categorías: $\geq 40$ años y < 40 años.

- Obesidad: se valoró a partir del índice de masa corporal (IMC). Se convirtió en dicotómica para el análisis univariado, en dos categorías: IMC $\geq 30$ y IMC $<30$.

- Estado físico: fue evaluado por el especialista de Anestesiología y Reanimación, se estimó según la clasificación de la American Society of Anesthesiologist (ASA)[27]. Se convirtió en dicotómico para el análisis univariado, en dos categorías: expuesto (Grado III-IV) y no expuesto (Grado I-II).

- Enfermedades asociadas (asma bronquial, hipertensión arterial e insuficiencia cardiaca).

Como variables para los factores de riesgo quirúrgico dependiente de la enfermedad, incluidas en el objetivo específico 2, se consideró:

- Tiempo transcurrido entre la hemodiálisis y la intervención. Se valoró a partir del tiempo transcurrido entre la última hemodiálisis y el momento de la intervención. Se convirtió en dicotómico para el análisis univariado, en dos categorías: expuesto (2 horas) y no expuesto (+ 2 horas).

- Tipo de anestesia. Fue valorado a partir del tipo de anestesia indicada al paciente según el proceder quirúrgico a realizar. Se convirtió en dicotómico para el análisis univariado, en dos categorías: expuesto (anestesia general) y no expuesto (anestesia regional).

\section{Resultados}

En la Tabla 1, se explica el resultado del análisis estadístico univariado realizado para identificar la posible asociación entre los factores de riesgo quirúrgico en función del enfermo, y la aparición de complicaciones anestésicas, cuando dichas variables hipotéticas se manifiestan aisladamente en la población estudiada.

En la Tabla 2 se relacionan los factores de riesgo quirúrgico dependiente de la enfermedad y su relación con la aparición de complicaciones anestésicas en enfermos renales crónicos intervenidos de urgencia.

En la Tabla 3 se constata la relación entre los factores de riesgo quirúrgico en función de la cirugía y la aparición de complicaciones anestésicas en enfermos renales crónicos sometidos a cirugía de urgencias. 


\begin{tabular}{|c|c|c|c|c|c|c|c|c|c|c|}
\hline & \multirow[t]{2}{*}{ FR } & \multirow{2}{*}{$\begin{array}{c}\text { Expues- } \\
\text { to } \\
\text { No }\end{array}$} & \multirow[b]{2}{*}{$\%$} & \multicolumn{2}{|c|}{ No expuestos } & \multicolumn{2}{|c|}{ Total } & \multirow[t]{2}{*}{$\mathbf{R R}$} & \multirow{2}{*}{$\begin{array}{c}\text { IC } \\
(95 \%)\end{array}$} & \multirow{2}{*}{$\begin{array}{l}\text { Probabi- } \\
\text { lidad }\end{array}$} \\
\hline & & & & No & $\%$ & No & $\%$ & & & \\
\hline Edad & $\begin{array}{l}\geq 40 \\
<40\end{array}$ & $\begin{array}{r}11 \\
4\end{array}$ & $\begin{array}{r}23,91 \\
8,69\end{array}$ & $\begin{array}{l}10 \\
21\end{array}$ & $\begin{array}{l}21,74 \\
45,65\end{array}$ & $\begin{array}{l}21 \\
25\end{array}$ & $\begin{array}{l}45,65 \\
54,35\end{array}$ & 5,77 & $\begin{array}{l}1,71- \\
24,86\end{array}$ & 0,0211 \\
\hline $\begin{array}{l}\text { O b e s i - } \\
\text { dad }\end{array}$ & $\begin{array}{l}\text { Sí } \\
\text { no }\end{array}$ & $\begin{array}{r}3 \\
12\end{array}$ & $\begin{array}{r}6,52 \\
26,09\end{array}$ & $\begin{array}{l}17 \\
14\end{array}$ & $\begin{array}{l}36,96 \\
30,43\end{array}$ & $\begin{array}{l}20 \\
26\end{array}$ & $\begin{array}{l}43,48 \\
56,52\end{array}$ & 0,20 & $\begin{array}{c}0,03- \\
0,76\end{array}$ & 0,0283 \\
\hline $\begin{array}{l}\text { Est a d o } \\
\text { físico }\end{array}$ & $\begin{array}{c}\text { III-IV } \\
\text { I-II }\end{array}$ & $\begin{array}{l}9 \\
6\end{array}$ & $\begin{array}{l}19,57 \\
13,04\end{array}$ & $\begin{array}{l}12 \\
19\end{array}$ & $\begin{array}{l}26,09 \\
41,30\end{array}$ & $\begin{array}{l}21 \\
25\end{array}$ & $\begin{array}{l}45,65 \\
54,35\end{array}$ & 2,37 & $\begin{array}{l}0,75- \\
8,10\end{array}$ & 0,2968 \\
\hline \multicolumn{11}{|c|}{ Enfermedades asociadas } \\
\hline HTA & $\begin{array}{l}\text { Sí } \\
\text { no }\end{array}$ & $\begin{array}{l}7 \\
8\end{array}$ & $\begin{array}{l}15,22 \\
17,39\end{array}$ & $\begin{array}{r}5 \\
26\end{array}$ & $\begin{array}{l}10,87 \\
56,52\end{array}$ & $\begin{array}{l}12 \\
34\end{array}$ & $\begin{array}{l}26,09 \\
73,91\end{array}$ & 4,55 & $\begin{array}{l}1,40- \\
14,35\end{array}$ & 0,0270 \\
\hline $\mathrm{Cl}$ & $\begin{array}{l}\text { Sí } \\
\text { no }\end{array}$ & $\begin{array}{r}3 \\
12\end{array}$ & $\begin{array}{r}6,52 \\
26,09\end{array}$ & $\begin{array}{r}1 \\
30\end{array}$ & $\begin{array}{r}2,17 \\
65,22\end{array}$ & $\begin{array}{r}4 \\
42\end{array}$ & $\begin{array}{r}8,69 \\
91,30\end{array}$ & 7,50 & $\begin{array}{l}1,35- \\
27,79\end{array}$ & 0,0584 \\
\hline IC & $\begin{array}{l}\text { Sí } \\
\text { no }\end{array}$ & $\begin{array}{r}5 \\
10\end{array}$ & $\begin{array}{l}10,87 \\
21,74\end{array}$ & $\begin{array}{r}3 \\
28\end{array}$ & $\begin{array}{r}6,52 \\
60,87\end{array}$ & $\begin{array}{r}8 \\
38\end{array}$ & $\begin{array}{l}17,39 \\
82,61\end{array}$ & 4,66 & $\begin{array}{l}1,25- \\
14,98\end{array}$ & 0,0472 \\
\hline $\begin{array}{l}\text { A s m a } \\
\text { B ro n - } \\
\text { quial }\end{array}$ & $\begin{array}{l}\text { Sí } \\
\text { no }\end{array}$ & $\begin{array}{r}2 \\
13\end{array}$ & $\begin{array}{r}4,35 \\
28,26\end{array}$ & $\begin{array}{r}1 \\
30\end{array}$ & $\begin{array}{r}2,17 \\
65,22\end{array}$ & $\begin{array}{r}3 \\
43\end{array}$ & $\begin{array}{r}6,52 \\
93,48\end{array}$ & 4,61 & $\begin{array}{l}0,50- \\
20,38\end{array}$ & 0,1931 \\
\hline
\end{tabular}

Fuente: Historia Clínica y planilla de vaciamiento de datos.

\begin{tabular}{|c|c|c|c|c|c|c|c|c|c|c|}
\hline & \multirow[t]{2}{*}{ FR } & \multicolumn{2}{|c|}{ Expuesto } & \multicolumn{2}{|c|}{ No expuestos } & \multicolumn{2}{|c|}{ Total } & \multirow[t]{2}{*}{$\mathbf{R} \mathbf{R}$} & \multirow{2}{*}{$\begin{array}{c}\text { IC } \\
(95 \%)\end{array}$} & \multirow{2}{*}{$\begin{array}{l}\text { Probabi- } \\
\text { lidad }\end{array}$} \\
\hline & & No & $\%$ & No & $\%$ & No & $\%$ & & & \\
\hline Estadio & $\begin{array}{l}1-2 \\
3-5\end{array}$ & $\begin{array}{r}1 \\
14\end{array}$ & $\begin{array}{r}2,17 \\
30,43\end{array}$ & $\begin{array}{r}5 \\
26\end{array}$ & $\begin{array}{l}10,87 \\
56,52\end{array}$ & $\begin{array}{r}6 \\
40\end{array}$ & $\begin{array}{l}13,04 \\
86,96\end{array}$ & 0,37 & $\begin{array}{c}0,008- \\
2,44\end{array}$ & 0,1014 \\
\hline $\begin{array}{l}\text { Medica- } \\
\text { m e n t o s } \\
\text { asociados }\end{array}$ & $\begin{array}{l}\text { Si } \\
\text { no }\end{array}$ & $\begin{array}{r}4 \\
11\end{array}$ & $\begin{array}{r}8,70 \\
23,91\end{array}$ & $\begin{array}{l}12 \\
19\end{array}$ & $\begin{array}{l}26,09 \\
41,30\end{array}$ & $\begin{array}{l}16 \\
30\end{array}$ & $\begin{array}{l}34,78 \\
65,22\end{array}$ & 0,57 & $\begin{array}{c}0,13- \\
1,94\end{array}$ & 0,6357 \\
\hline $\begin{array}{l}\text { T i e m p o } \\
\text { transcurrido } \\
\text { Hemodiáli- } \\
\text { sis-Cirugía }\end{array}$ & $\begin{array}{l}-2 h \\
+2 h\end{array}$ & $\begin{array}{r}3 \\
12\end{array}$ & $\begin{array}{r}6,52 \\
26,09\end{array}$ & $\begin{array}{r}1 \\
30\end{array}$ & $\begin{array}{r}2,17 \\
65,22\end{array}$ & $\begin{array}{r}4 \\
42\end{array}$ & $\begin{array}{r}8,70 \\
91,30\end{array}$ & 7,50 & $\begin{array}{l}1,35- \\
27,7\end{array}$ & 0,0584 \\
\hline \multicolumn{11}{|c|}{ Causas de la enfermedad renal crónica } \\
\hline HTA & $\begin{array}{c}\text { Si } \\
\text { no }\end{array}$ & $\begin{array}{l}6 \\
9\end{array}$ & $\begin{array}{l}13,04 \\
19,57\end{array}$ & $\begin{array}{r}4 \\
27\end{array}$ & $\begin{array}{r}8,70 \\
58,70\end{array}$ & $\begin{array}{l}10 \\
36\end{array}$ & $\begin{array}{l}21,74 \\
78,26\end{array}$ & 4,50 & $\begin{array}{l}1,31- \\
14,15\end{array}$ & 0,0397 \\
\hline DM & $\begin{array}{c}\text { Si } \\
\text { no }\end{array}$ & $\begin{array}{r}5 \\
10\end{array}$ & $\begin{array}{l}10,87 \\
21,74\end{array}$ & $\begin{array}{r}8 \\
23\end{array}$ & $\begin{array}{l}17,39 \\
50,00\end{array}$ & $\begin{array}{l}13 \\
33\end{array}$ & $\begin{array}{l}28,26 \\
71,74\end{array}$ & 1,43 & $\begin{array}{c}0,38- \\
4,61\end{array}$ & 0,1014 \\
\hline $\begin{array}{l}\text { Glomerulo- } \\
\text { patía }\end{array}$ & $\begin{array}{l}\text { Si } \\
\text { no }\end{array}$ & $\begin{array}{r}3 \\
12\end{array}$ & $\begin{array}{r}6,52 \\
26,09\end{array}$ & $\begin{array}{r}5 \\
26\end{array}$ & $\begin{array}{l}10,87 \\
56,52\end{array}$ & $\begin{array}{r}8 \\
38\end{array}$ & $\begin{array}{l}17,39 \\
82,61\end{array}$ & 1,30 & $\begin{array}{c}0,23- \\
4,81\end{array}$ & 0,0462 \\
\hline $\begin{array}{l}\text { Riñones po- } \\
\text { liquísticos }\end{array}$ & $\begin{array}{l}\text { Si } \\
\text { no }\end{array}$ & $\begin{array}{r}4 \\
11\end{array}$ & $\begin{array}{r}8,70 \\
23,91\end{array}$ & $\begin{array}{r}9 \\
22\end{array}$ & $\begin{array}{l}19,57 \\
47,83\end{array}$ & $\begin{array}{l}13 \\
33\end{array}$ & $\begin{array}{l}28,26 \\
71,74\end{array}$ & 0,88 & $\begin{array}{l}0,20- \\
2,99\end{array}$ & 0,0323 \\
\hline
\end{tabular}

Fuente: Historia Clínica y planilla de vaciamiento de datos. 


\begin{tabular}{l} 
Tabla 3. Factores de riesgo quirúrgico en función de la cirugía. Hospital Carlos Manuel de Céspedes. \\
Análisis univariado. 2014- $\mathbf{2 0 1 7}$ \\
\hline
\end{tabular}

Fuente: Historia Clínica y planilla de vaciamiento de datos.

\begin{tabular}{|c|c|c|c|c|c|c|}
\hline \multirow[b]{2}{*}{ Variables } & \multirow[t]{2}{*}{ B } & \multirow[t]{2}{*}{ SE } & \multirow[t]{2}{*}{ p } & \multirow[t]{2}{*}{ HR } & \multicolumn{2}{|c|}{ Intervalo de Confianza del $95 \%$} \\
\hline & & & & & Inferior & Superior \\
\hline Hipertensión arterial & 0,508 & 0,157 & 0,001 & 1,661 & 1,221 & 2,261 \\
\hline Cardiopatía isquémica & 0,493 & 0,168 & 0,003 & 1,638 & 1,117 & 2,278 \\
\hline Duración de la cirugía & 0,483 & 0,127 & 0,000 & 1,620 & 1,263 & 2,079 \\
\hline $\begin{array}{l}\text { Tiempo entre la hemo- } \\
\text { diálisis y la cirugía }\end{array}$ & 0,371 & 0,153 & 0,025 & 1,376 & 1,037 & 1,799 \\
\hline Edad & 0,288 & 0,102 & 0,040 & 1,313 & 1,666 & 1,991 \\
\hline Insuficiencia cardiaca & 0,259 & 0,114 & 0,023 & 1,272 & 1,617 & 1,965 \\
\hline Tipo de anestesia & 0,246 & 0,168 & 0,006 & 1,224 & 1,501 & 1,869 \\
\hline
\end{tabular}

Fuente: Historia Clínica y planilla de vaciamiento de datos.

\section{Discusión}

En pacientes con enfermedad renal, la agresión quirúrgica puede dañar, aún más, la función renal al producirse trastornos hidroelectrolíticos y del equilibrio ácido-base, el conocimiento de la enfermedad renal y la valoración de los factores de riesgo permite tomar medidas de conducción que impida o disminuya el daño[13].

En este estudio la edad igual o mayor de 40 años constituyó un factor de riesgo; lo que puede estar relacionado con los factores degenerativos propios del proceso de envejecimiento que afectan el sistema nervioso central, el aparato locomotor, el aparato digestivo y el respiratorio, así como a la función renal[8],[9],[11].

Los resultados obtenidos en la presente investigación, donde en los pacientes en que existe la asociación de enfermedad renal crónica con hipertensión arterial, cardiopatía isquémica o insuficiencia cardiaca, se incrementa el riesgo de complicaciones anestésicas[14],[15].

En la presente investigación la hipertensión arterial como causa de la enfermedad renal estuvo asociada a la aparición de complicaciones anestésicas.

En la presente investigación la glomerulopatía como causa de enfermedad renal crónica se asoció a la aparición de complicaciones anestésicas, resultados que coinciden con planteamientos de otros autores[2],[7],[26].

\section{Conclusiones}

1. La edad, la hipertensión arterial, la cardiopatía isquémica y la insuficiencia cardiaca fueron los factores de riesgo quirúrgico en función del enfermo asociados a complicaciones. 
2. El tiempo transcurrido entre la hemodiálisis y la intervención menor de 2 horas, y cuando la insuficiencia renal fue secundaria a hipertensión arterial y glomerulopatía se constituyeron en factores de riesgo quirúrgico dependiente de la enfermedad.

3. Los factores de riesgo quirúrgico en función de la cirugía fueron el tiempo de la cirugía menor de 2 horas y el empleo de anestesia general.

\section{Referencias}

1. Gámez Jiménez AM, Montell Hernández OA, Ruano Quintero $\checkmark$, Alfonso de León JA, Hay de la Puente Zoto M. Enfermedad renal crónica en el adulto mayor. Rev Méd Electrón [Internet]. 2013 Jul-Ago [citado: 25 de abril 2015];35(4). Disponible en: http://www.revmatanzas.sld. cu/revista\%20medica/ano\%20 2013/vol4\%202013/tema01.htm

2. Inserra F, De la Llave $G$, Alpino M, Castagna R, De la Fuente I, Dorado $E$, et al. Relevamiento de factores de riesgo y de enfermedad renal en familiares de pacientes en diálisis. Medicina [Internet]. 2007 [citado 25 de abril 2015];67(1):8-18. Disponible en: http://www.scielo.org.ar/ scielo.php?script=sci_arttext\&pid =S0025-76802007000100002

3. Soriano Cabrera S. Definición y clasificación de los estadios de la enfermedad renal crónica. Prevalencia. Claves para el diagnóstico precoz. Factores de riesgo de enfermedad renal crónica. Nefrología [Internet]. 2008 [citado 25 de abril 2015];24(Supl. 6). Disponible en: http://www.revistanefrologia.com/revistas/P7-E237/ P7-E237-S141-A3100.pdf

4. De Francisco M, Aguilera L, Fuster V. Enfermedad cardiovascular, enfermedad renal y otras enfermedades crónicas. Nefrología [Internet]. 2009 [citado 25 de abril 2015];29(1):6-9. Disponible en: http://www.revistanefrologia. $\mathrm{com} /$ modules. .php? name=articul os\&idarticulo $=223 \&$ idlangart $=E S$

5. De Francisco ÁL, De la Cruz JJ,
Cases A, De la Figuera M, Egocheaga MI, Górriz JI. Prevalencia de insuficiencia renal en Centros de Atención Primaria en España: estudio EROCAP. Nefrología [Internet]. 2007 [citado 25 de abril 2015];27(3). Disponible en: http://www.revistanefrologia. com/revistas/P1-E258/P1-E258S132-A4548.pdf

6. Otero A, Abelleira A, Gayoso P. Enfermedad renal crónica oculta (ERCO), y factores de riesgo vascular (FRV) asociados. Estudio epidemiológico. Nefrología [Internet]. 2005 [citado 25 de abril 2015];25(3). Disponible en: http://www.revistanefrologia. com/revistas/P1-E242/P1-E242S132-A3212.pdf

7. González Cárdenas VH, Guillermo Vargas J, Enrique Echeverri J, Díaz SM, Mena Méndez Y. Valoración preanestésica en el paciente con enfermedad renal crónica (énfasis en riesgo cardiovascular). Revcolomb anestesiol.2013;41(2):139-145.

8. Cordero Escobar I. Anestesiología. Criterios y tendencias actuales. La Habana: Editorial Ciencias Médicas; 2013.

9. Dávila Cabo de Villa, E. Anestesiología Clínica. Segunda edición La Habana: Editorial Ciencias Médicas. 2014.

10. Pérez Oliva Díaz JF, Herrera Valdés R, Almaguer López M. ¿Cómo mejorar el manejo de la enfermedad renal crónica? Consideraciones y recomendaciones prácticas. Rev Habanera de Ciencias Méd [Internet]. 2008 [citado 25 de abril 2015];7(1):122. Disponible en: http://scielo. sld.cu/scielo.php?script=sci_ arttext\&pid=S1729519X2008000100009

11. Ojeda González José Julio. Elementos predictores en la evaluación del riesgo anestésico por el anestesiólogo. Rev cuba anestesiolreanim [revista en la Internet]. 2013 Jun [citado 2015 Abr 14]; 12(2): 158-168. Disponible en: http://scielo. sld.cu/scielo.php?script=sci_ arttext\&pid=S1726$67182013000200007 \& \operatorname{lng}=e s$

12. National Kidney Foundation. K/ DOQI clinical practice guidelines for chronic kidney disease: evaluation, classification, and stratification. Am J Kidney Dis. 2002 Feb;39(2 Suppl 1):S1-266.

13. Abd El Bagi ME, Almutairi BM, Alsolamy SJ. Imaging of nontraumatic acute abdominal pain in adults presenting to the emergency department. Saudi Med J. 2016 Mar;37(3):324-7.

14. Vargas-Trujillo C. Anestesia en el paciente con hipertensión arterial sistémica. Revista Mexicana de Anestesiología. 2015 AprJun;38 Supl. 1:S71-80.

15. San Román JA. Comentarios a la guía de práctica clínica de la ESC/ESA 2014 sobre cirugía no cardiaca: evaluación y manejo cardiovascular. Rev Esp Cardiol. 2014;67(12):980-5.

16. Kristensen SD, Knuuti J, Saraste A, Anker S, Bøtker HE, Hert SD, et al.; Authors/Task Force Members. 2014 ESC/ESA Guidelines on non-cardiac surgery: cardiovascular assessment and management: The Joint Task Force on non-cardiac surgery: 
cardiovascular assessment and management of the European Society of Cardiology (ESC) and the European Society of Anaesthesiology (ESA). Eur Heart J. 2014 Sep;35(35):2383-431.

17. James PA, Oparil S, Carter BL, Cushman WC, Dennison-Himmelfarb C, Handler J, et al. 2014 evidence-based guideline for the management of high blood pressure in adults: report from the panel members appointed to the Eighth Joint National Committee (JNC 8). JAMA. 2014 Feb;311(5):507-20.

18. Sierra P, Galcerán JM, Sabaté $S$, Martínez-Amenós A, Castaño J, Gi A. Documento de consenso sobre hipertensión arterial y anestesia de las Sociedades Catalanas de Anestesiología e Hipertensión Arterial. Hipertens Riesgo Vasc. 2009;26(5):218-28.

19. Pannell LM, Reyes EM, Underwood SR. Cardiac risk assessment before non-cardiac surgery. Eur Heart J Cardiovasc Imaging. 2013 Apr;14(4):316-22

20. Upshaw J, Kiernan MS. Preoperative cardiac risk assessment for noncardiac surgery in patients with heart failure. Curr Heart Fail Rep. 2013 Jun;10(2):147-56.

21. Grupo de Trabajo Conjunto sobre cirugía no cardiaca: evaluación y manejo cardiovascular de la Sociedad Europea de Cardiología (ESC) y la EuropeanSociety of Anesthesiology(ESA). Guía de práctica clínica de la ESC/ESA 2014 sobre cirugía no cardiaca: evaluación y manejo cardiovascular. Rev Esp Cardiol. 2014;67(12):1052.e1-e43.

22. Fleisher LA, Beckman JA, Brown KA, Calkins H, Chaikof EL, Fleischmann KE, et al.; American College of Cardiology Foundation/ American Heart Association Task Force on Practice Guidelines; American Society of Echocardiography; American Society of Nu- clear Cardiology; Heart Rhythm Society; Society of Cardiovascular Anesthesiologists; Society for Cardiovascular Angiography and Interventions; Society for Vascular Medicine; Society for Vascular Surgery. 2009 ACCF/AHA focused update on perioperative beta blockade incorporated into the ACC/AHA 2007 guidelines on perioperative cardiovascular evaluation and care for noncardiac surgery. J Am Coll Cardiol. 2009 Nov; 54(22):e13-118.

23. Gómez-Huelgas R, MartínezCastelao A, Artola S, Górriz $J L$, Menéndez E. Documento de Consenso sobre el tratamiento de la diabetes tipo 2 en el paciente con enfermedad renal crónica. Nefrologia 2014;34(1):1-12. Disponible en: http://www.revista-nefrologia. com/revistas/P1-E565/P1-E565S4493-A12369.pdf [Accedido: 30/12/2017]

24. Rydén L, Grant PJ, Anker SD, Berne C, Cosentino F, Danchin $\mathrm{N}$, et al.; Document Reviewers. ESC Guidelines on diabetes, pre-diabetes, and cardiovascular diseases developed in collaboration with the EASD: the Task Force on diabetes, pre-diabetes, and cardiovascular diseases of the European Society of Cardiology (ESC) and developed in collaboration with the European Association for the Study of Diabetes (EASD). Eur Heart J. 2013 Oct;34(39):3035-87.

25. Pottie K, Jaramillo A, Lewin G, Dickinson J, Bell N, Brauer P, et al.; Canadian Task Force on Preventive Health Care. Recommendations on screening for type 2 diabetes in adults. CMAJ. 2012 Oct;184(15):1687-96.

26. Fernández-García JO, CervantesMaldonado HE, Zarazúa-Juárez M, Reyes-Rodríguez DM, Castellanos-Olivares A. Protocolo anestésico para trasplante renal del Hospital de Especialidades del Centro Médico Nacional Siglo XXI. Vol. 40. No. 3 Julioseptiembre 2017, pp 176-189.

27. Jojoa JA, Bravo C, Vallejo C. Clasificación práctica de la enfermedad renal crónica 2016: una propuesta. Repert Med Cir. 2016 Jul-Sep;25(3):192-6.

28. Martínez-Castelao A, Górriz JL, Bover J, Segura-de la Morena J, Cebollada J, Escalada J, et al. Documento de consenso para la detección y manejo de la enfermedad renal crónica. Nefrología [Internet]. 2014 [citado $20 \mathrm{Jul}$ 2017];34(2):243-262. Disponible en: http://scielo.isciii.es/pdf/nefrologia/v34n2/documento_consenso.pdf

29. Uchikawa T, Shimano M, Inden $Y$, Murohara T. Serum albumin levels predict clinical outcomes in chronic kidney disease (CKD) patients undergoing cardiac resynchronization therapy. InternMed [Internet]. 2014 [citado 20 Jul 2017];53(6):555-61. Disponible en: http://www.ncbi.nlm.nih. gov/pubmed/24633024

30. KDIGO 2012 Clinical Practice Guideline for the Evaluation and Management of Chronic Kidney Disease. Summary of Recommendation Statements. [Accedido:30/12/2013].Kidney Inter-national Supplements (2013) 2, 419-28. Disponibleen: http://www.nature.com/kisup/ journal/v3/n1/full/kisup201277a. html

31. Bell JS, Blacker N, Leblanc VT, Alderman CP, Phillips A, Rowett $D$, et al. Prescribing for older people with chronic renal impairment. Aust Fam Physician. 2013 Jan-Feb;42(1-2):24-8.

32. Jones DR, Lee HT. Surgery in the patient with renal dysfunction. Med Clin North Am. 2009 Sep;93(5):1083-93.

33. Reich DL, Bennett-Guerrero $E$, Bodian CA, Hossain S, Winfree 
W, Krol M. Intraoperative tachycardia and hypertension are independently associated with adverse outcome in noncardiac surgery of long duration. Anesth Analg. 2002 Aug;95(2):273-7.

34. Clarke-Pearson DL, Dodge RK, Synan I, McClelland RC, Maxwell
GL. Venous thromboembolism prophylaxis: patients at high risk to fail intermittent pneumatic compression. Obstet Gynecol. 2003 Jan;101(1):157-63.

35. Wagener G, Brentjens TE. Anesthetic concerns in patients presenting with renal failure. Anesthesiol Clin. 2010 Mar;28(1):39-54.

36. Meersch, M; Schmidt, C; Zarbock, A.Patient with chronic renal failure undergoing surgery. Current Opinion in Anesthesiology: June 2016 - Volume 29 Issue 3 - p 413-420. 